\title{
A New Approach to measure Phonon Dispersion Relation (PDR) by Raman Scattering and a Downbending Observation in Diamond PDR
}

Shu-Lin Zhang, ${ }^{\text {a }}$ Lei Xia, Weihua Chen, D. Y. Li, Wanyu Li and Juan He

State Key Laboratory for Artificial Microstructure and Mesoscopic Physics, School of Physics, Peking University, Beijing 100871, China

\begin{abstract}
The phonon dispersion relation (PDR), i.e., the dependence of phonon frequency $\omega$ on its wavevector $q, \omega(q)$, was measured traditionally by inelastic neutron scattering (INS) or inelastic X-ray scattering (IXS). A new approach to measure PDR by Raman scattering (RS) of nanostructures was proposed and applied to observe the longitudinal optical (LO) PDR of diamond successfully. Due to the higher resolution and accuracy of $\omega$ and $q$ in RS, a clear downbending feature of $\omega$ with increasing $q$ away from the Brillouin zoon center was observed for the first time.

The validity of the new approach has been confirmed also by the appearing of the downward bending in PDR, which is originally measured by traditional high-resolution IXS experiment.

The downbending feature may give us a clue for deep understanding of the interactions occur in diamond, while the overbending feature observed by INS and IXS has been attributed to strong effective second-nearest-neighbor forces.
\end{abstract}

Keywords:

A. Nanostructures;

C. Diamond;

D. Phonon dispersion relation;

E. Raman spectroscopy.

\section{Introduction}

The phonon dispersion relation (PDR), $\omega(q)$, describes the dependence of the phonon frequency $\omega$ on its wavevector $q$ and is an important foundation of solid state physics. The PDR is derived theoretically by the lattice dynamics and experimentally by the inelastic scattering (IS). For IS, the frequencies $\omega_{i}, \omega_{s}, \omega_{q}$ and the wavevectors $\boldsymbol{k}_{i}, \boldsymbol{k}_{s}, \boldsymbol{q}$ for the incident wave, scattered wave and phonon itself satisfy the conservation law of energy $E(=\hbar \omega)$ and momentum $\boldsymbol{P}(=\hbar \boldsymbol{k}$ or $\hbar \boldsymbol{q})$, i.e.,

$$
\omega_{i}-\omega_{s}=\omega_{q}
$$

a) Electronic mail: slzhang@pku.edu.cn 


$$
\boldsymbol{k}_{i}-\boldsymbol{k}_{s}=\boldsymbol{q}
$$

Thus, PDR can be derived by an IS. However, in the IS of light (i.e., Raman scattering, $\mathrm{RS}$ ), there is a so-called Raman selection rule of $q=0$, i.e., the phonons with $q \neq 0$ are Raman inactive [1]. As a result, the PDR is impossible to be deduced by RS and is traditionally measured by inelastic X-ray scattering (IXS) [2, 3] or inelastic neutron scattering (INS) [4, 5]. However, the IXS and INS is not as good as RS in terms of resolution, precision and accuracy of frequency. For example, the resolution, accuracy and precision of frequency are $64 \mathrm{~cm}^{-1}, 12 \mathrm{~cm}^{-1}$ and $2.5 \mathrm{~cm}^{-1}$, respectively, even though for high resolution IXE [2]. In contrast, the resolution, accuracy and precision of frequency can easily reach to $0.2 \mathrm{~cm}^{-1}, 1.5 \mathrm{~cm}^{-1}$ and $0.25 \mathrm{~cm}^{-1}$, respectively in RS. Therefore the PDR derived by RS should have an obvious advantage in resolution, precision and accuracy of frequencies and has hence attracted attention.

The RS spectra of nanostructures have been a subject since early 1980s and many significant theoretical, experimental and review articles [6-10] have been published in this field. It is well known that for nanostructures with a finite size must possess a finite size effect (FSE), by which one of the induced results is the loss of the translational symmetry and relaxation of the momentum $\boldsymbol{P}$ (i.e., wavevector $k$ or $q$ ) conservation. That results in a consequence that the selection rule of phonon wavevector $q=0$ in $\mathrm{RS}$ is relaxed and the wavevector of Raman active phonons can be non-zero, indicating a possibility to deduce PDR by RS.

It is well known that the uncertainty principle is a basic principle in quantum mechanics, which claims that the standard deviation of size $L$ and wavevector $q$, $\Delta L$ and $\Delta q$, satisfies

$$
\Delta L \Delta q \geq 1 / 2
$$

The uncertainty principle does not play an actual role in a macroscopic medium with large $L$. However, due to the value of $L$ and corresponding $\Delta L$ of nanostructures being very small, the uncertainty principle may have a real impact in nanostructures. Thus, based on the uncertainty principle, the $\Delta q$ of a nanostructure can be finite and the deduced value from by $\Delta L$ as well as the wavevector $q$ of nanostructures with bulk wavevector $q_{b}$ will be $q=q_{b}+\Delta q$. Since $q_{b}=0$ as $q_{b}$ being at the Brillouin zoon center, we will have $q=\Delta q$ for nanostructures. That implies that $q$ of nanostructures can be obtained by its $\Delta L$ directly. Moreover, if the relative standard deviation $\Delta L / L$ is smaller and equivalent for all of nanostructure samples being used, the dependence of $\omega$ on $q$ for nanostructures can be deduced by various sizes of nanostructures. Additionally, if the crystal structure of nanostructures is the same as that of corresponding bulk material, the measured dependence of $\omega$ on $q$ of the nanostructures can be considered as PDR. The above statement implies that a non-traditional approach to measure PDR by RS is valid. 
In this paper the suggested non-traditional approach to measure PDR is confirmed by derivation of the longitudinal optical (LO) PDR of diamond by Raman spectra of nano-crystalline diamond (NCD).

\section{Experimental methods}

It is has been mentioned that the nano-diamonds get smaller, the non-intrinsic structures and defects may appear [11, 12]. Therefore it is very important that the nano-diamond samples used to measure the PDR of diamond should be the diamond structure and have uniformed size.

All samples named by NCD-U used in this work were prepared by a self-organization approach, of which NCD-U1 was prepared by the detonation method and NCD-U2-U5 were prepared by chemical vapor deposition. It is well known that the samples prepared by self-organization approach has a same crystalline structure with bulks and no impurity and distortion to be produced [13, 14]. Therefore the samples used are fitting the requirement of samples.

The transmission electron microscopy (TEM) and scanning electron microscopy (SEM) images of samples were obtained from FEI Tecnai TEMF 30 and FEI Nano SEM 430, respectively. The transparent electron microscopy (TEM) images of NCD-U samples are shown in Fig 1. From visual inspection of the TEM image of over 100 clearly delineated particles of NCD-U, the average size $L_{\mathrm{av}}$ and standard deviation $\Delta L_{\mathrm{av}}$ were obtained and listed in Table 1 . The relative standard deviation $(\Delta L / L)_{\mathrm{av}}$ of NCD-U samples has a similar value of 0.09 to 0.11 , implying the nano-crystalline diamonds used have different but uniformed sizes definitely.

The Raman spectral measurements were performed by using Renishaw Invia Raman microprobes in backscattering geometry at room temperature. The instrument resolution and spectral accuracy of the Raman microprobes are about $1.5 \mathrm{~cm}^{-1}$ and 0.5 $\mathrm{cm}^{-1}$, respectively. In the treatment of measured Raman spectra, only the optical phonon with SP3 were used, which guarantees the PDR to be belong to the optical phonon

Besides, from Fig 1 we can also see that the NCD particles in samples have no shared orientation, indicating that the measured PDR can't be recognized to be along a certain principal axis. In this work we suppose that the measured PDR is along an average direction of the three principal axis, i.e., the $\Gamma_{L}$ direction.

\section{Results and discussion}

The observed and fitted (with a Lorentzian line shape) Raman spectra exited by a $514.5 \mathrm{~nm}$ laser for samples NCD-U are shown in Fig 2 (a) in solid and dashed lines, respectively. From Fig 2 (a) we can see that the fitted spectra exhibit two peaks. It is 
well known that the phonons of $L O$ and $T O$ of diamond degenerate at the Brillouin zoon center but split while leaving the center. Due to FSE the observed phonons of nanostructures could be away from the Brillouin zoon center, i.e., $L O$ and TO phonons could be observed simultaneously. Thus we assign the two peaks to TO and $L O$ phonons for the samples with size being smaller than $6.61 \mathrm{~nm}$ and $L O$ and $D$ phonons for a sample of the largest size, of which the $D$ mode is originated from disorder structures in sample. The fitted RS frequencies, $\omega$ of $T O$ and $L O$, were listed in Table 1. From Table 1 we can also see that the Raman frequency separation between $L O$ and $T O$ increases monotonously with decreasing of the sample size, conforming the sample possessing FSE and the measured RS spectra being definitely originated from NCD.

The wavevectors $q$ were deduced from $\Delta L_{\mathrm{av}}$ based on formula (3) and the corresponding reduced wavevectors $q_{\mathrm{r}}\left(=q_{/} q_{\max } ; q_{\max }=\pi / a, a=0.35668 \mathrm{~nm}\right.$ is the lattice constant of diamond) were listed Table 1

Based on the date in Table 1, the Raman frequencies of $L O$ phonon $\omega_{\mathrm{L}}$ with reduced wavevector $q_{\mathrm{r}}$ are drawn with solid triangle in Fig 3 (a). In Fig 3 (a), one can see a downward bending feature of $\omega_{\mathrm{L}}$ with increasing of $q_{\mathrm{r}}$ just leaving the Brillouin zoon center. This feature is not found in the diamond PDR observed by traditional IXS $[2,3]$ and INS $[4,5]$ and thus may reflect that the present $\omega(q)$ derived by RS may not be reliability. To check the reliability we performed a comparison with independent RS experiments for UCD-U samples.

The first comparison is by using a single-size NCD sample. Such kind of samples quoted were the single NCD particles with size of 90 and $35 \mathrm{~nm}$ [15], for which we labeled them as NCD-S1 and NCD-S2, respectively. The sizes of NCD-S1 and NCD-S2 are just in between the sizes of NCD-1 (581.6nm) and NCD-2 $(6.61 \mathrm{~nm})$. To deduce $q$ we selected $\Delta L$ of NCD-S being $0.1 \times L$, as $L$ is measured for a single particle and must possess a high accuracy. The $\omega_{L}$ of NCD-S with their reduced wavevectors $q_{r}$ are listed in Table 1 and marked in Fig 3 (a) with red solid square. From Fig 3 (a), we can see clearly that the data of the NCD-S samples are located just between NCD-1 and NCD-2 in the down part of the downward bending, indicating the reliability of the downward bending feature and giving a positive confirmation on the PDR measured for NCD-U samples.

The second comparison is performed by a size-distributed NCD sample named as NCD-D. The SEM image of NCD-D is shown in Fig 1 (g) and the sizes of the NCD particles in NCD-D are 3-120 nm. The RS spectra of NCD-D were exited by 325, 488, 515, 633, 785 and $1064 \mathrm{~nm}$ lasers and shown in Fig 2 (b) in which the solid and dashed lines are the observed and fitted (with a Lorentzian) Raman spectra, respectively. The Raman frequencies, $\omega$, of the fitted Raman peaks were listed in Table 2. Fig 2 (c) is the photo-luminescence (PL) spectrum of the NCD-D sample. 
The PL spectrum indicates that the Raman spectra shown in Fig 2 (b) are indeed the resonant RS spectra. Thus, the size of nano-particles in NCD-D can be selected by the resonant size selection effect (RSSE) according to the wavelength $\lambda_{\text {ex }}$ (i.e., excited energy $\left.E_{\mathrm{ex}}\right)$ of the excitation lasers [16, 17].

For the selection of the nano-particle size $L_{s e}$ by RSSE, a dependence of sample size $L$ on electron band gap energy, $E_{\text {gap }}$, is needed. For this requirement, a theoretical $L$ dependence of $E_{\text {gap }}$ for nano-diamond (ND) clusters showing in Fig 4 of Ref 18 was quoted. However, in Fig 4 of Ref 18, the $E_{\text {gap }}$ range of 5.5-8.0 $\mathrm{eV}$ doesn't cover the energy range of the exciting laser $\lambda_{\mathrm{ex}}, 1.211-3.815 \mathrm{eV}$. Thus, it is impossible to select out the size of ND particles, $L_{\mathrm{ex}}$, in NCD-D, directly from using Fig 4 of Ref 18 . To overcome this difficulty, firstly, we figured out an average gradient $G$ of the change of $L$ with $E_{\text {gap }}$ in the range of small $E_{\text {gap }}$ in Fig $4^{[18]}$, obtaining $G=1.556 \mathrm{~nm} / \mathrm{eV}$. Secondly, we found a fiducial ND particle in NCD-D, of which the size $L_{\text {fid }}$ could be determined based on an independent and reliable information. For this, we found that the Raman frequency $\omega$ of $1925 \mathrm{~cm}^{-1}$ appears simultaneously for NCD-U4 with 6.30 $\mathrm{nm}$ listed in Table 2 and in NCD-D exited by $633 \mathrm{~nm}$ laser $(=1.958 \mathrm{eV})$ listed in Table 2. It is thus reasonable to determine the particle selected by $633 \mathrm{~nm}$ laser being the fiducial ND with $L_{\text {fid }}=6.30 \mathrm{~nm}$. Thirdly, after getting the difference of a certain excited energy relative to $1.958 \mathrm{eV}, E_{\mathrm{dif}}$, the size of this excited particle is determined to be $L_{s e}=L_{\mathrm{fid}}+\left(G \times E_{\mathrm{dif}}\right)$. Moreover, we made $\Delta L_{\mathrm{se}}$ to be $0.1 \times L_{\mathrm{se}}$, as $L$ is selected by RSSE. $L_{\mathrm{ex}}, \Delta L_{\mathrm{se}},(\Delta L / L)_{\mathrm{se}}$ and $q_{\mathrm{r}}$ of NCD particles in NCD-D were listed in Table 2. From Table 2, it can be seen that two Raman peaks appeared in the fitted spectra exhibit same characteristics with those in samples NCD-U, meaning that the two peaks appeared in samples NCD-D must have the same properties of NCD-U.

The dependence of $\omega_{\mathrm{L}}$ on $q_{\mathrm{r}}$ listed in Table 2 were plotted in Figure 3 (b) from which we can see that with increasing of $q_{\mathrm{r}}$ from the center of Brillouin zoon the value of $\omega_{\mathrm{L}}$ decreases from $1931.4 \mathrm{~cm}^{-1}$ to the smallest value of $1925.0 \mathrm{~cm}^{-1}$ and then increases to the largest value of $1335.0 \mathrm{~cm}^{-1}$. That again indicates the appearing of downward bending feature. Since the largest value of $1335.0 \mathrm{~cm}^{-1}$ surpasses that at the center of Brillouin zoon, $1332 \mathrm{~cm}^{-1}$, the overbending feature observed in INS ${ }^{2,3}$ and IXS ${ }^{4,5}$ appears too. Therefore, the reliability of the LO PDR by RS shown in Fig 3 (a) obtains a further approval.

It is well known that a stronger test of the validity of a new procedure must show that the feature appearing in the new procedure, such as downward bending here, should also appear in traditional approaches. For this kind of checking, we quote a diamond PDR measured by a high-resolution IXS ${ }^{2}$. This PDR has some specialties for comparing with the PDR measured by RS. First, it is from the highest resolution IXS measurement, as far as we know. Secondly, the authors of Ref 2 mentioned that the observed PDR is only along one direction of $\Delta$, meaning that it is a good sample to 
compare with the RS measured PDR with only one average direction of $q$. Thirdly, the PDR shown in Fig 3 of Ref 2 has a highest resolution in IXS and INS measurements. Based on the third specialty we have easily figured out that the measured frequencies $\omega$ at $q_{\mathrm{r}}=0.0,0.1$ and 0.2 are $1332 \mathrm{~cm}^{-1}, 1325 \mathrm{~cm}^{-1}$ and $1335 \mathrm{~cm}^{-1}$, respectively. That shows in fact that there is also a downward bending feature before the appearing of the overbending. Especially, the smallest $\omega_{L}$ in the downward bending is $1325 \mathrm{~cm}^{-1}$ at $q_{\mathrm{r}}=0.1$, being basically equivalent to $1325.4 \mathrm{~cm}^{-1}$ at $q_{\mathrm{r}}=$ 0.0834 in the PDR measured for the NCD-U samples and $1325.0 \mathrm{~cm}^{-1}$ at $q_{\mathrm{r}}=0.0901$ in the PDR measured for the NCD-D sample. Obviously, those mentioned above gives the new RS approach a conclusive confirmation.

\section{Conclusions}

We propose a non-traditional approach by using Raman scattering (RS) of nanostructures with different sizes to measure the phonon dispersion relation (PDR). By using this new technique we measured the PDR of diamond in the reduced wavevector range $q_{\mathrm{r}}$ of $0.0-0.2$. Thanks to the high resolution in frequency and wavevector of phonons in the measurement of RS, the PDR deduced by different RS experiments for different kinds of nano-crystalline diamond samples are all showing a downward bending feature just away from the center of Brillouin zoon, which was not observed before. In fact, the downward bending feature is also found in the PDR of diamond deduced by highest resolution inelastic X-ray scattering. Obviously all of the above facts prove the solid reliability of the new approach and the new-observed downward bending feature.

Moreover, the downward bending feature may give us a clue in deep understanding of the interactions presented in diamond, as the universally accepted overbending feature in the diamond PDR has been attributed to the strong effective second nearest-neighbor forces neglected before.

\section{Acknowledgment}

The authors acknowledge the support from the National Basic Research Program of China under grants No. 2014CB846001, 2009CB929403, 2007CB815602 and the NSF of China under Grant No. 51102003. We thank Prof. Jian Chen, Prof. C-L Cheng and Dongguang Chuangli Lapping Tec. Co. for providing samples. The authors want to thank Prof. K.T. Yue and Prof. Weikun Ge for their technical assistance.

\section{References}

[1] S. L. Zhang, Raman spectroscopy and its Application in Nanostructures, John 
Wiley Sons Ltd, Chichester, United Kingdom, 2012.

[2] M. Schwoerer-Bo“hning, A.T. Macrander, and D.A. Arms, Phys. Rev. Lett. 80 (1998) 5572.

[3] E. Burkel, Inelastic Scattering of X-Rays with Very High Energy Resolution, Springer Tracts in Modern Physics, 125 (1991) 61.

[4] J. Kulda, H. Kainzmaier, D. Strauch, B. Dorner, M. Lorenzen, and M. Krisch, Phys. Rev. B, 66 (2002) 241202.

[5] J. Kulda, B. Dorner, B. Roessli, H. Sterner, R. Bauer, Th. May, K.Karch, P. Pavone, and D. Strauch, Solid State Commun. 99 (1996) 799.

[6] Campbell H, Fauchet PM. Solid State Commun. 58 (1986) 739.

[7] Roca E, Giner CT, Cardona M. Phys. Rev., B, 49 (1994) 13704.

[8] Giner CT, Debernardi A, Cardona M, Proupin EM, Ekimov AI. Phys. Rev., B, 57 (1998) 4664.

[9] Zi J, Zhang K, Xie X. Phys. Rev., B, 55 (1997) 9263.

[10] Akhilesh K. Arora, M. Rajalakshmi, T. R. Ravindran and V. Sivasubramanian, J. Raman Spectrosc. 38 (2007) 604.

[11] Prawer et al, Chemical Physics Letters, 332（2000）93.

[12] Osswald et al, Phys. Rev. B, 80(2009) 075419.

[13] Krsttaced N, Ledentsov N. N and Grandmann et al, Electrinics Lett, 30(1994)1416.

[14] Nözel R and Ploog K, Adv Mater, 5(1993)22.[15] K. W. Sun, J. Y. Wang, and T. Y. Ko, Appl Phys Lett, 92 (2008) 153115.

[15] K. W. Sun, J. Y. Wang, and T. Y. Ko, Appl Phys Lett, 92 (2008) 153115.

[16] S. L. Zhang, W. Ding, Y. Yan, J. Qu, Bibo Li, Le-Yu Li, K. T. Yue, and D.P. Yu, Appl. Phys. Lett. 81 (2002) 4446.

[17] A. M. Rao, E. Richter, S. Bandow, B. Chase, P. C. Eklund, K. A.Williams, S. Fang, K. R. Subbaswamy, M. Menon, A. Thess, R. E.Smalley, G. Dresselhaus, and M. S. Dresselhaus, Science, 275 (1997) 187.

[18] D.A. Areshkin, O.A. Shenderova, S.P. Adiga, D.W. Brenner, Diamond \& Related Materials, 13 (2004) 1826. 
Fig. 1. TEM images of nano-crystalline diamond samples of NCD-U1 (a), NCD-U2 (b), NCD-U3 (c), NCD-U4 (d), NCD-U5 (e), NCD-U6 (f) and SEM image of NCD-D (g).

Fig. 2. The observed (in solid line) and fitted (in dashed line) Raman scattering (RS) spectra exited by $514.5 \mathrm{~nm}$ laser for size-uniformed nano-crystalline diamond samples NCD-U (a) and exited by 325, 488, 515, 633, 785 and $1064 \mathrm{~nm}$ lasers for size-distributed NCD-U sample (b). The photo-luminescence (PL) spectrum of NCD-U are shown in (c). Where $L O, T O$, and $D$ refer to the longitudinal optical, transverse optical and disorder structure phonons, respectively.

Fig. 3. The dependence of Raman frequencies of $L O$ phonon $\omega_{\mathrm{L}}$ with reduced wavevector $q_{\mathrm{r}}$ shown in (a) for nano-crystalline diamond (NCD) samples with size-uniformed NCD-U (drawn with black solid square) and with single particle NCD-S (drawn with red solid square) and in (b) for size-distributed sample NCD-D (drawn with solid triangle). 


\section{Table 1}

The measured Raman frequencies $\omega$ excited by exiting wavelength $\lambda_{\mathrm{ex}}=514.5 \mathrm{~nm}$ laser for nano-crystalline diamond (NCD) with uniformed size NCD-U. LO, TO and $D$ label longitudinal optical, transverse optical and disorder phonons, respectively. $L_{\mathrm{av}}$, $\Delta L_{\mathrm{av}}$ and $\Delta L_{\mathrm{av}} / L_{\mathrm{av}}$ label the size, standard deviation and relative standard deviation, respectively. $q_{\mathrm{r}}$ is the reduced wavevector of $q\left({ }_{=} q_{/} q_{\max } ; q_{\max }=\pi / a, a=0.35668 \mathrm{~nm}\right.$ is the lattice constant of diamond).

\begin{tabular}{|c|c|c|c|c|c|c|c|c|}
\hline \multicolumn{2}{|c|}{ Sample } & NCD-U1 & NCD-S1 & UCD-S2 & NCD-U2 & NCD-U3 & NCD-U4 & NCD-U5 \\
\hline \multirow{3}{*}{ Size } & \multirow{3}{*}{$\begin{array}{c}L_{\mathrm{av}}(\mathrm{nm}) \\
\Delta L_{\mathrm{av}}(\mathrm{nm}) \\
(\Delta L / L)_{\mathrm{av}}\end{array}$} & 581.6 & 90 & 35 & 6.61 & 6.30 & 5.55 & 3.95 \\
\hline & & 52.7 & $\sim 9,0$ & $\sim 3.5$ & 0.75 & 0.68 & 0.54 & 0.40 \\
\hline & & 0.09 & $\sim 0.10$ & $\sim 0.10$ & 0.11 & 0.11 & 0.10 & 0.10 \\
\hline \multirow{4}{*}{$\begin{array}{c}\text { Raman } \\
\text { frequency } \\
\quad\left(\mathrm{cm}^{-1}\right)\end{array}$} & \multirow{4}{*}{$\begin{array}{c}\omega_{L O} \\
\omega_{T O} \\
\omega_{L O-T O} \\
\omega_{D}\end{array}$} & 1332.5 & 1329 & 1325 & 1324.3 & 1325.0 & 1326.3 & 1330.0 \\
\hline & & I & I & I & 1309.6 & 1310.1 & 1311.2 & 1313.7 \\
\hline & & / & I & I & 14.7 & 14.9 & 15.1 & 16.3 \\
\hline & & 1359.0 & I & I & 1 & / & I & 1 \\
\hline \multicolumn{2}{|c|}{$q_{\mathrm{r}}$} & 0.00176 & 0.00630 & 0.0162 & 0.0756 & 0.0834 & 0.105 & 0.142 \\
\hline
\end{tabular}

\section{Table 2}

The measured Raman frequencies $\omega$ excited by exiting wavelength by exiting wavelength $\lambda_{\mathrm{ex}}=325,488,515,633,785$ and $1064 \mathrm{~nm}$ lasers for size-distributed sample NCD-D. $L O$ and TO label longitudinal optical and transverse optical phonons, respectively. $L_{\mathrm{se}}, \Delta L_{\mathrm{se}}$ and $\Delta L_{\mathrm{se}} / L_{\mathrm{se}}$ label the size, standard deviation and relative standard deviation. $q_{\mathrm{r}}$ is the reduced wavevector of $q\left({ }_{=} q_{/} q_{\max } ; q_{\max }=\pi / a, a=0.35668\right.$ $\mathrm{nm}$ is the lattice constant of diamond).

\begin{tabular}{|c|c|c|c|c|c|c|c|}
\hline \multirow{2}{*}{$\begin{array}{c}\text { Excited } \\
\text { wavelength }\end{array}$} & $\lambda_{\mathrm{ex}}(\mathrm{nm})$ & 1024 & 785 & 633 & 515 & 488 & 325 \\
\cline { 3 - 8 } & $\lambda_{\mathrm{ex}}(\mathrm{eV})$ & 1.211 & 1.580 & 1.959 & 2.405 & 2.538 & 3.815 \\
\hline \multirow{2}{*}{$\begin{array}{c}\text { Raman } \\
\text { frequency } \\
\left(\mathrm{cm}^{-1}\right)\end{array}$} & $\omega_{L O}$ & 1331.4 & 1328.2 & 1325.4 & 1329.8 & 1330.1 & 1335.5 \\
\cline { 3 - 8 } & $\omega_{T O}$ & $/$ & 1326.2 & 1323.2 & 1327.5 & 1327.6 & 1331.6 \\
\cline { 3 - 8 } & $\omega_{L O-T O}$ & $/$ & 2.0 & 2.2 & 2.3 & 2.5 & 3.9 \\
\hline \multirow{2}{*}{ Size } & $L_{\text {se }}(\mathrm{nm})$ & 7.464 & 6.890 & 6.30 & 5.906 & 5.399 & 3.412 \\
\cline { 3 - 8 } & $\Delta L_{\mathrm{se}}(\mathrm{nm})$ & 0.746 & 0.689 & 0.630 & 0.591 & 0.540 & 0.341 \\
\cline { 3 - 8 } & $(\Delta L / L)_{\mathrm{se}}$ & 0.10 & 0.10 & 0.10 & 0.10 & 0.10 & 0.10 \\
\hline \multicolumn{2}{|c|}{$q_{r}$} & 0.0761 & 0.0824 & 0.0901 & 0.0961 & 0.105 & 0.166 \\
\hline
\end{tabular}




\section{Tables}

\section{Table 1}

The measured Raman frequencies $\omega$ excited by exiting wavelength $\lambda_{\mathrm{ex}}=514.5 \mathrm{~nm}$ laser for nano-crystalline diamond (NCD) with uniformed size NCD-U. LO, TO and $D$ label longitudinal optical, transverse optical and disorder phonons, respectively. $L_{\mathrm{av}}$, $\Delta L_{\mathrm{av}}$ and $\Delta L_{\mathrm{av}} / L_{\mathrm{av}}$ label the size, standard deviation and relative standard deviation, respectively. $q_{\mathrm{r}}$ is the reduced wavevector of $q\left(=q_{/} q_{\max } ; q_{\max }=\pi / a, a=0.35668 \mathrm{~nm}\right.$ is the lattice constant of diamond).

\begin{tabular}{|c|c|c|c|c|c|c|c|c|}
\hline \multicolumn{2}{|c|}{ Sample } & NCD-U1 & NCD-S1 & UCD-S2 & NCD-U2 & NCD-U3 & NCD-U4 & NCD-U5 \\
\hline \multirow{3}{*}{ Size } & \multirow{3}{*}{$\begin{array}{c}L_{\mathrm{av}}(\mathrm{nm}) \\
\Delta L_{\mathrm{av}}(\mathrm{nm}) \\
(\Delta L / L)_{\mathrm{av}}\end{array}$} & 581.6 & 90 & 35 & 6.61 & 6.30 & 5.55 & 3.95 \\
\hline & & 52.7 & $\sim 9,0$ & $\sim 3.5$ & 0.75 & 0.68 & 0.54 & 0.40 \\
\hline & & 0.09 & $\sim 0.10$ & $\sim 0.10$ & 0.11 & 0.11 & 0.10 & 0.10 \\
\hline \multirow{4}{*}{$\begin{array}{c}\text { Raman } \\
\text { frequency } \\
\qquad\left(\mathrm{cm}^{-1}\right)\end{array}$} & \multirow{4}{*}{$\begin{array}{c}\omega_{L O} \\
\omega_{T O} \\
\omega_{L O-T O} \\
\omega_{D}\end{array}$} & 1332.5 & 1329 & 1325 & 1324.3 & 1325.0 & 1326.3 & 1330.0 \\
\hline & & 1 & I & I & 1309.6 & 1310.1 & 1311.2 & 1313.7 \\
\hline & & 1 & I & I & 14.7 & 14.9 & 15.1 & 16.3 \\
\hline & & 1359.0 & I & 1 & / & 1 & I & I \\
\hline \multicolumn{2}{|c|}{$q_{\mathrm{r}}$} & 0.00176 & 0.00630 & 0.0162 & 0.0756 & 0.0834 & 0.105 & 0.142 \\
\hline
\end{tabular}

\section{Table 2}

The measured Raman frequencies $\omega$ excited by exiting wavelength by exiting wavelength $\lambda_{\mathrm{ex}}=325,488,515,633,785$ and $1064 \mathrm{~nm}$ lasers for size-distributed sample NCD-D. $L O$ and TO label longitudinal optical and transverse optical phonons, respectively. $L_{\mathrm{se}}, \Delta L_{\mathrm{se}}$ and $\Delta L_{\mathrm{se}} / L_{\mathrm{se}}$ label the size, standard deviation and relative standard deviation. $q_{\mathrm{r}}$ is the reduced wavevector of $q\left({ }_{=} q_{/} q_{\max }, q_{\max }=\pi / a, a=0.35668\right.$ $\mathrm{nm}$ is the lattice constant of diamond).

\begin{tabular}{|c|c|c|c|c|c|c|c|}
\hline \multirow{2}{*}{$\begin{array}{c}\text { Excited } \\
\text { wavelength }\end{array}$} & $\lambda_{\mathrm{ex}}(\mathrm{nm})$ & 1024 & 785 & 633 & 515 & 488 & 325 \\
\cline { 3 - 8 } & $\lambda_{\mathrm{ex}}(\mathrm{eV})$ & 1.211 & 1.580 & 1.959 & 2.405 & 2.538 & 3.815 \\
\hline \multirow{2}{*}{$\begin{array}{c}\text { Raman } \\
\text { frequency } \\
\left(\mathrm{cm}^{-1}\right)\end{array}$} & $\omega_{L O}$ & 1331.4 & 1328.2 & 1325.4 & 1329.8 & 1330.1 & 1335.5 \\
\cline { 3 - 8 } & $\omega_{\text {TO }}$ & $/$ & 1326.2 & 1323.2 & 1327.5 & 1327.6 & 1331.6 \\
\cline { 3 - 8 } & $\omega_{\text {LO-TO }}$ & $/$ & 2.0 & 2.2 & 2.3 & 2.5 & 3.9 \\
\hline \multirow{2}{*}{ Size } & $L_{\text {se }}(\mathrm{nm})$ & 7.464 & 6.890 & 6.30 & 5.906 & 5.399 & 3.412 \\
\cline { 3 - 8 } & $\Delta L_{\text {se }}(\mathrm{nm})$ & 0.746 & 0.689 & 0.630 & 0.591 & 0.540 & 0.341 \\
\cline { 3 - 8 } & $(\Delta L / L)_{\text {se }}$ & 0.10 & 0.10 & 0.10 & 0.10 & 0.10 & 0.10 \\
\hline \multicolumn{2}{|c|}{$q_{r}$} & 0.0761 & 0.0824 & 0.0901 & 0.0961 & 0.105 & 0.166 \\
\hline
\end{tabular}



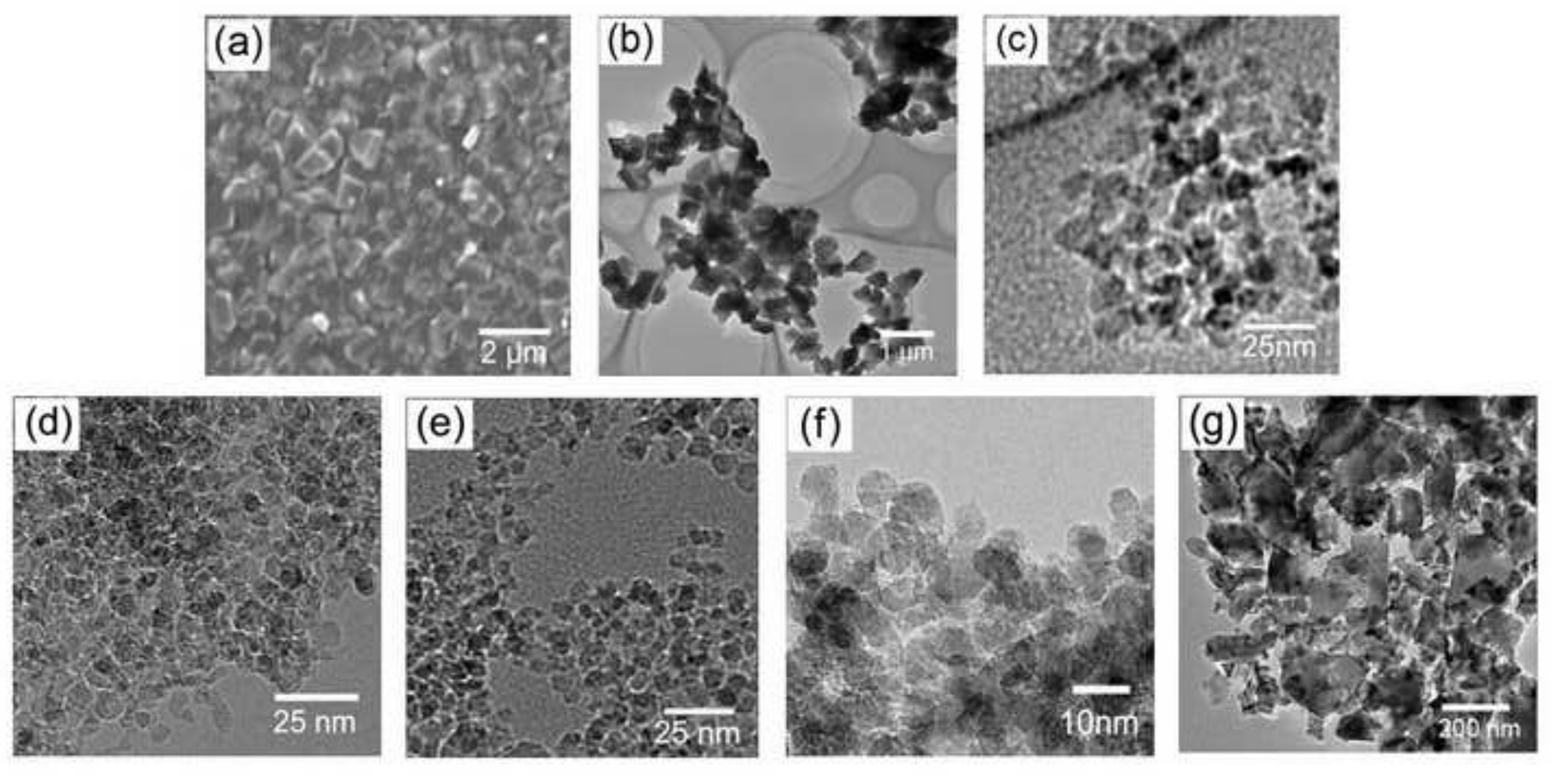


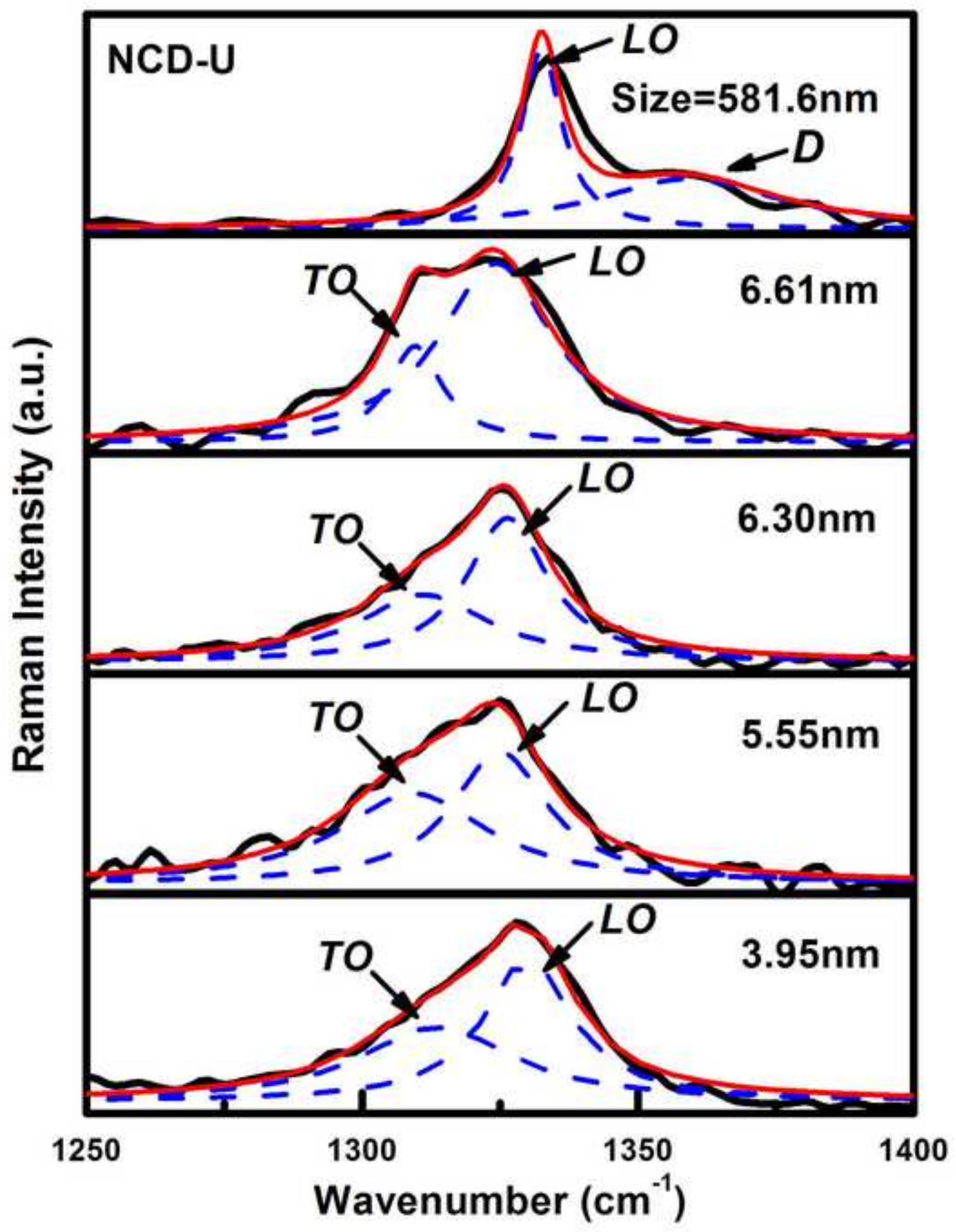

(a) 


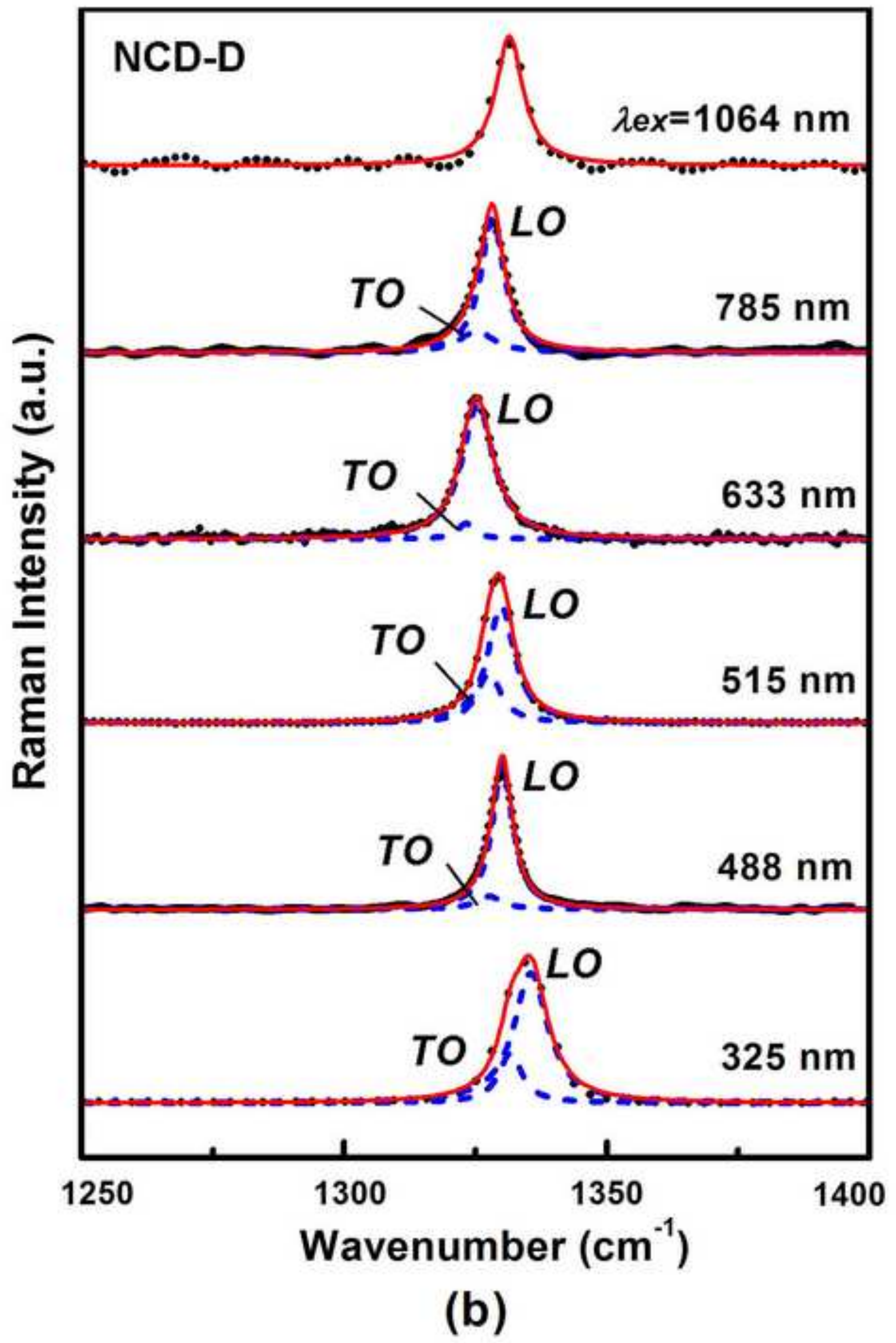




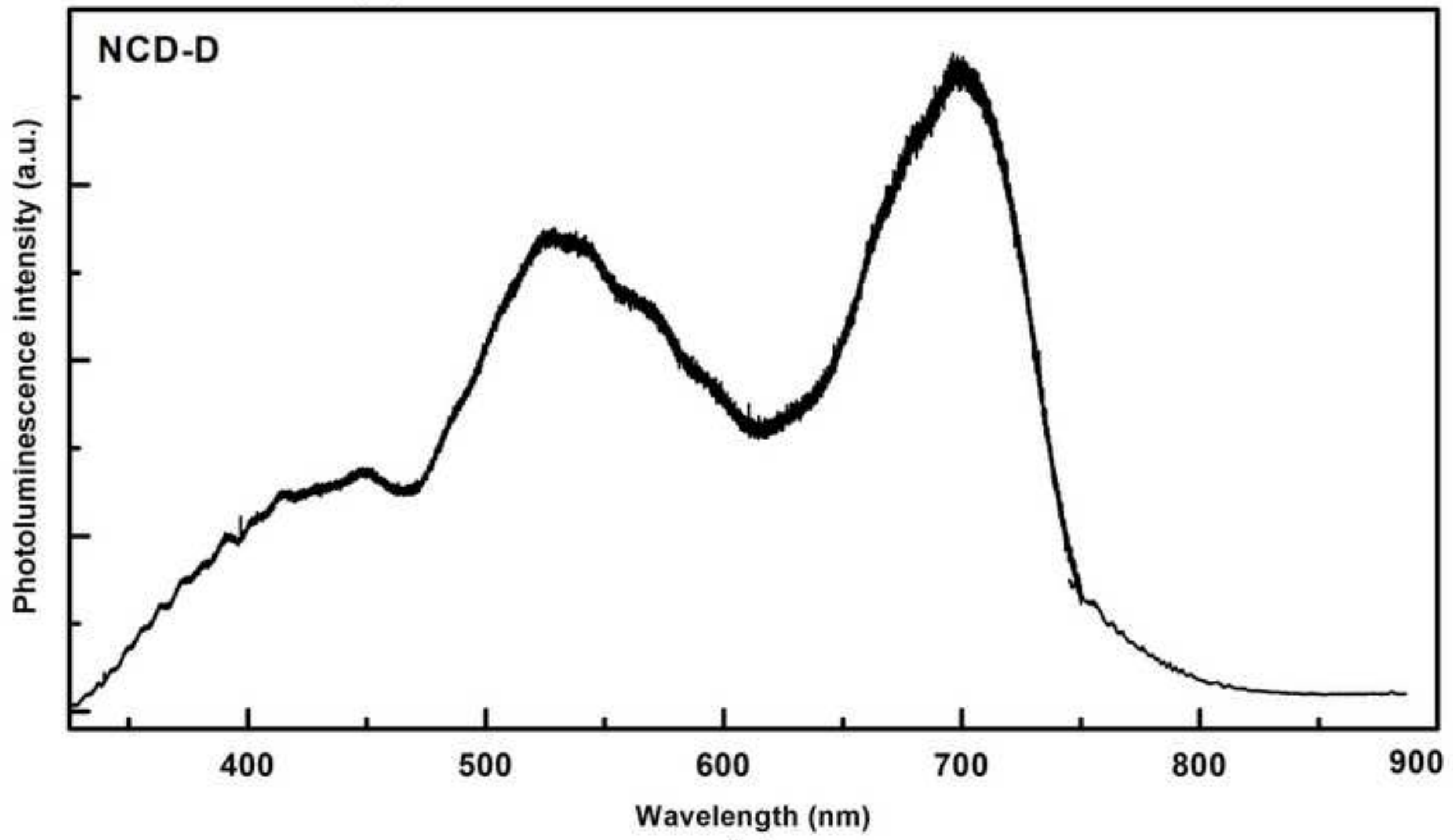

(c) 


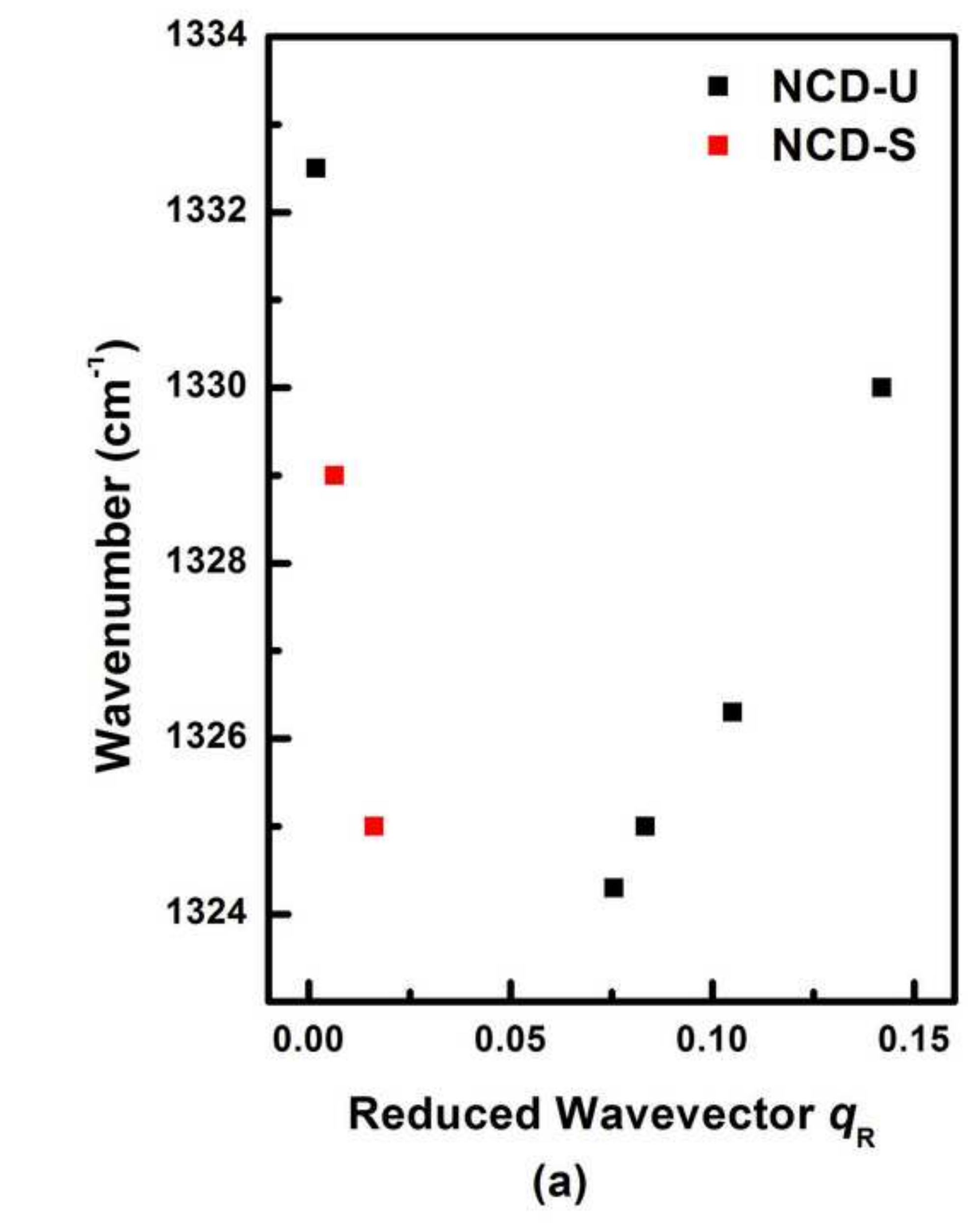

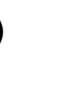
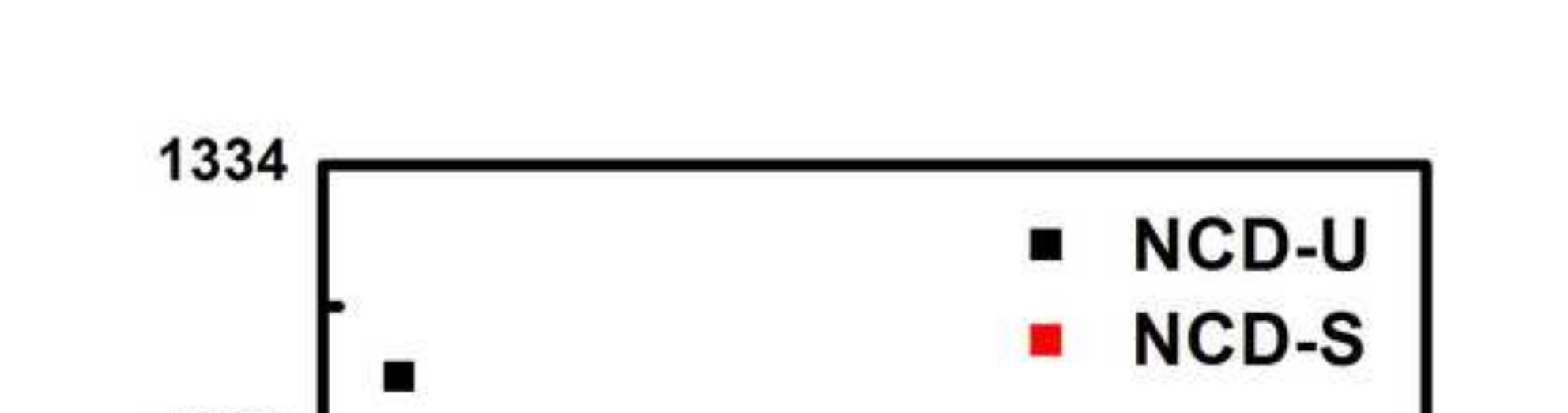


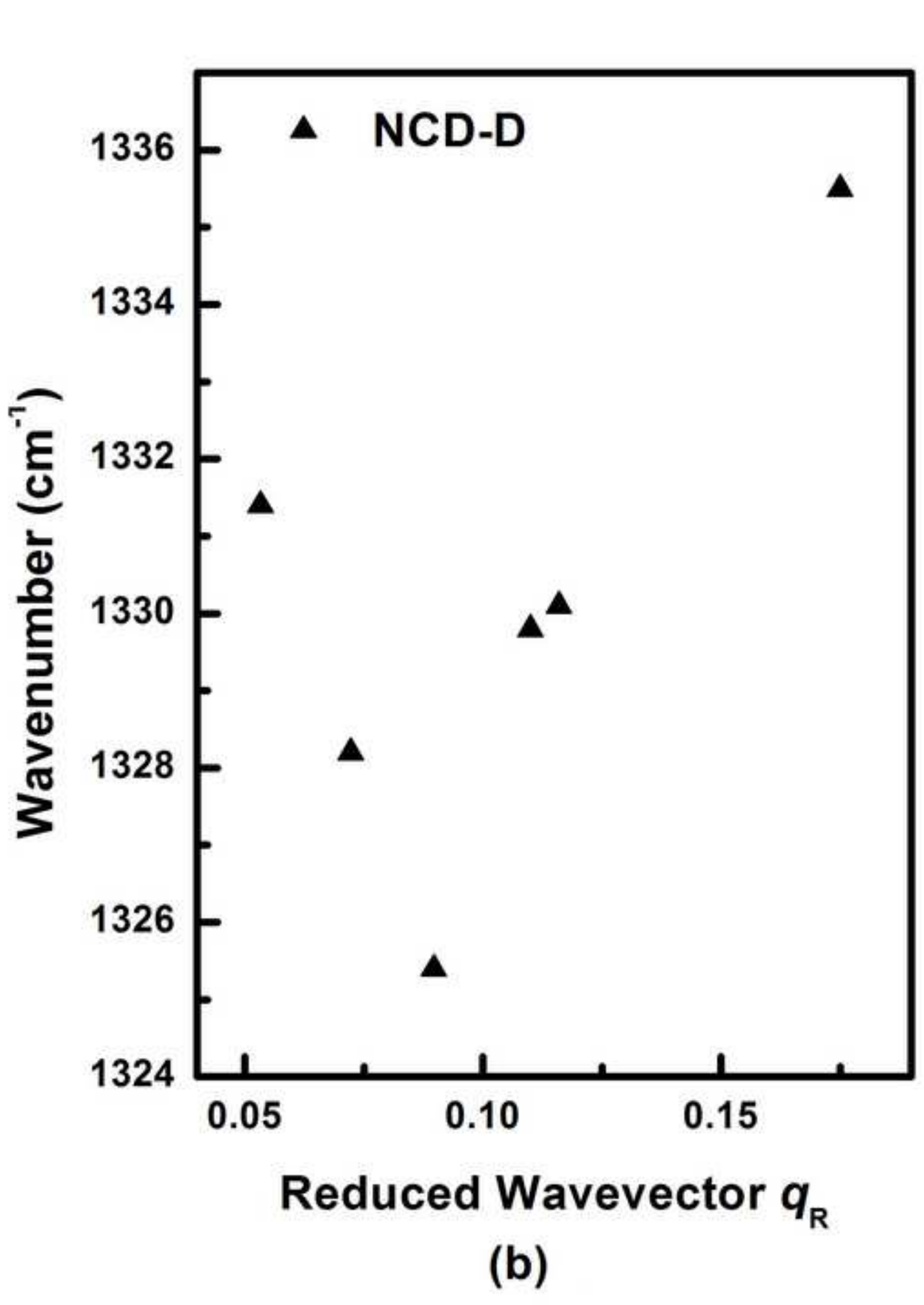

)

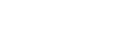
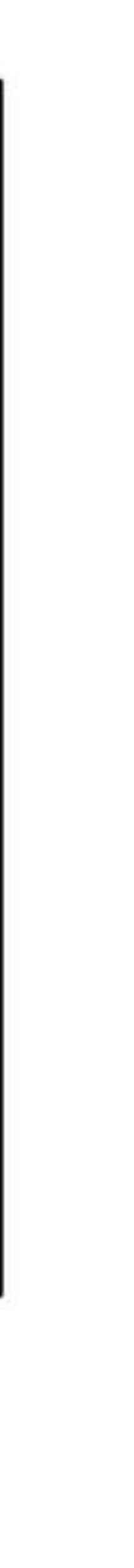